\title{
Current progress and future direction in the treatment for hemophilia
}

\author{
Midori Shima $^{1}$
}

Received: 4 November 2019 / Revised: 19 November 2019 / Accepted: 26 November 2019 / Published online: 7 December 2019

(c) Japanese Society of Hematology 2019

Hemophilia A and B are the most common of the severe congenital coagulopathies. These disorders are attributable to deficiencies of FVIII and FIX, and are associated with various types of recurrent bleeding. The main principles of treatment for hemophilia depend on regular prophylaxis using FVIII or FIX concentrates. Several unmet needs remain in current hemophilia treatment, however. These include the requirement for repeated intra-venous infusions, the development of inhibitors, and fluctuations together with low trough levels of clotting factor activity. Furthermore, completely preventing the progression of hemophilic arthropathy remains an unresolved challenge.

Non-clotting factor therapeutics have been developed to overcome these clinical difficulties, Emicizumab, a humanized recombinant bispecific antibody mimicking activated FVIII (FVIIIa), is one such product that has been developed in Japan, and which has been approved in more than 60 countries. In this context, Kitazawa et al. have described the development and biochemical properties of emicizumab [1], and a Phase 1 study of single subcutaneous injections in healthy individuals was performed in 2012 [2]. The half-life of emicizumab was found to be around 30 days. In addition, prophylactic efficacy after weekly subcutaneous injections was also explored in a complementary study that included a total of 18 hemophilia A patients with and without inhibitors in three dose cohorts [3]. The treated annual bleeding rate (ABR) was markedly reduced and the effect was shown to be maintained for up to 2.7 years in an extension study [4]. There were no significant drug-related adverse events other than local skin reactions at sites of injection.

The safety and prophylactic efficacy of emicizumab have been examined in a series of international Phase 3 studies involving a total of 400 subjects (HAVEN 1-4 [5-8]

Midori Shima

mshima@naramed-u.ac.jp

1 Department of Pediatrics, Nara Medical University, 840 Shijo-cho, Kashihara City, Nara, Japan and HOHOEMI studies [9]). The reductions in ABR were remarkable, and the proportion of patients with zero treated bleeds was around $60 \%$, even in regimens involving treatment at intervals of 4 weeks. Two thrombo-emobolic (TE) events and three incidents of thrombotic microangiopathy (TMA) were reported in the HAVEN 1 study. High and frequent doses of APCC were used in all patients. None of the patients that received only rFVIIa developed TE or TMA. Hence, the recommendation was to use rFVIIa as the first line option for breakthrough bleeding during emicizumab prophylaxis. Three of the 400 cases in the HAVEN 1 and 2 studies developed neutralizing ADA. The HOHOEMI study included one previously untreated patient (PUP) and the results demonstrated that the treated ABR and the zero treated ratio were similar to those in the HAVEN studies [9]. Flexible dosing (weekly, every 2 and 4 weeks) may be beneficial for hemophilia A patients of all ages. TE/TMA should be carefully monitored during hemostatic therapy. Recently, longer term efficacy and safety in all HAVEN studies $(n=400)$ were examined after a median observation term of 83 weeks. Accordingly, the treated ABR over consecutive 24-week treatment intervals decreased, and the rate of zero bleeds increased over time. At $73-114$ weeks $(n=114)$, the zero-bleed rate was $88.6 \%$. Furthermore, no additional drugrelated ADE were observed [10]. These extended results provide strong evidence for the clinical utility of emicizumab. Several practical issues for emicizumab prophylaxis remain, however, including requirements for ITI and laboratory monitoring of hemostatic treatment.

In normal hemostasis, coagulation function is critically regulated by various natural inhibitors, including anti-thrombin (AT), protein $\mathrm{C}$, protein $\mathrm{S}$, and tissue factor pathway inhibitor (TFPI).

TFPI is a potent inhibitor at the initiation of coagulation, and is triggered by FVIIa/TF complexes. TFPI consists of an $\mathrm{N}$-terminal region, three Kunitz domains (K1, K2 and $\mathrm{K} 3$ ), and a $\mathrm{C}$-terminal region. $\mathrm{K} 1$ and $\mathrm{K} 2$ are responsible for inhibition of FVIIa and FXa, respectively. 
The biochemical properties of TFPI are summarized elegantly by Chowdary [11]. Neutralization of TFPI can restore disordered thrombin generation, and therefore, TFPI inhibition is an attractive concept for hemostatic control in hemophilia. The first TFPI inhibitor devised for this purpose was a nucleic acid aptamer, BAX 499, which binds TFPI specifically [12]. Phase 1 studies revealed, however, that the number of bleeding episodes increased in the highest dose cohort. This appeared to be due to a 25 -fold increase in the plasma concentration of full-length TFPI [13], and resulted in discontinuation of the clinical trial.

The second class of therapeutics based on TFPI inhibition involves anti-TFPI monoclonal antibodies, of which three are currently under clinical investigation. Concizumab is a humanized recombinant monoclonal IgG4 antibody that recognizes the K2 domain of TFPI. The antibody consolidates thrombin generation by neutralizing TFPI mediated inhibition of FVIIa, and FXa. In a Phase 1 clinical trial, named EXPLORER [14], concizumab exhibited a dose-dependent, non-linear pharmacokinetic profile, and thrombin generation correlated well with concizumab exposure and the level of free TFPI.

In subsequent trials (eg. EXPLORER 3) $[15,16]$ there was a trend towards a lower ABR at higher concentrations of concizumab, especially over $100 \mathrm{ng} / \mathrm{mL}$ Inverse D-dimer and prothrombin fragment $1+2$ levels have been reported, and depressed fibrinogen levels have been observed at the highest dose in the dose-escalation studies and in the intravenous cohort at the highest doses (1000 or $3000 \mu \mathrm{g} / \mathrm{kg}$ ). One healthy subject developed thrombophlebitis. The findings of the early EXPLORER trials have been concisely summarized by the same authors [11]. More recently, the results of a Phase 2 study were published [17]. In this trial concizumab was administered by daily subcutaneous injections. Estimated ABRs in hemophilia A/B with inhibitor and hemophilia A were 3.0/5.9 and 7.0, respectively. Three patients had non-neutralizing ADA with no observed clinical effect. PF-06741086 is another monoclonal antibody against the TFPI-recognizing K2 domain [18]. A Phase 1 study in healthy individuals administered subcutaneously and with intravenous infusions demonstrated the pharmacological efficacy of this agent. A Phase 2 multi-dose study in hemophilia A and B patients without inhibitors is now under way. A third antibody, designated BAY-1093884, recognizes the K1 and K2 domains [19], and a Phase 2 study is also ongoing. Although information on the long-term safety and efficacy of anti-TFPI antibodies is limited and there are concerns about thrombotic events in various pathological circumstances, the available evidence indicates that these antibodies may offer advantages in the treatment of hemophilia B patients with inhibitors, for which treatment is, at present, extremely difficult.
Gene therapy may provide much longer term benefits after a single treatment procedure, and the clinical application of this technology in hemophilia has been anticipated for over a decade. The review by Ohmori [20] discusses the potential impact of gene therapy especially in developing countries where the availability of coagulation factor concentrates is restricted. Strategies for gene therapy in hemophilia involve direct intravenous administration of a viral vector carrying a therapeutic gene in vivo. Several relevant virus vectors have been developed, and in the most of current clinical trials in hemophilia, adeno-associated virus (AAV) has been used to transduce FVIII or FIX genes directly into liver cells. There are several advantages of AAV compared to other vectors. For example, recombinant AAV vectors do not undergo site-specific integration in host DNA, but mainly remain episomal in the nucleus of transduced cells. In addition, the pathogenicity and immunogenicity of the AAV vectors are markedly lower than with the wild-type virus, and the AAV vectors have the ability to transfer the target gene into quiescent cells.

The first generation AAV2 serotype used for early clinical trials in patients with hemophilia B was administered by intramuscular injection [21]. The treatment effects were not satisfactory, however, due to low levels of FIX expression. In further clinical trials, elevated therapeutic levels of FIX were achieved using higher doses of AAV2 $\left(2 \times 10^{12} \mathrm{vg} / \mathrm{kg}\right)$ [22] These levels of FIX declined, however, linked especially with an increase of liver transaminase activity. The underlying mechanism appeared to be associated with destruction of transduced hepatocytes, mediated by cell-mediated immunity involving the major histocompatibility complex (MHC) class 1 and $\mathrm{CD}^{+}{ }^{+} \mathrm{T}$-cells targeting antigens of the AAV capsid, causing both the decline in FIX and the transient increase in transaminase activity.

Later vectors, AAV5 and AAV8, made it possible to transduce target genes to the liver efficiently by portal vein or intravenous administration. Long-term expression of clinically meaningful levels of FIX and safety were confirmed for up to 3.5 years by Nathwani et al. [23] in a phase $1 / 2$ clinical trial using AAV8. Liver dysfunction occurred in the high-dose vector group, but this was well controlled by corticosteroid therapy.

A newer AAV serotype (Spark-100) developed by Spark Therapeutics Inc has been included in a Phase 1/2 study of hemophilia $\mathrm{B}$, and demonstrated promising results after intravenous administration [24]. The vector incorporated mutant FIX (FIX Padua) with eightfold activity compared to wild type FIX, which made it possible to obtain $30 \mathrm{IU} /$ dL FIX activity at a much lower vector dose $\left(5 \times 10^{11} \mathrm{vg} /\right.$ $\mathrm{kg})$. Furthermore, only two patients needed treatment with corticosteroids to control cytotoxic T Cell- mediated liver dysfunction. A phase 3 clinical trial using AAV8 with FIX Padua (Shire/Takeda) is imminent. 
Gene therapy for hemophilia A has advanced more slowly than that for hemophilia B. These delays have centered mainly on low expression due to the large size of FVIII gene that is poorly incorporated into the AAV vector. However, BioMarin Pharmaceutical Inc. have recently reported promising results in a phase $1 / 2$ study of hemophilia A using AAV5 vector harboring B domain-deleted FVIII at a low dose $\left(6 \times 10^{12} \mathrm{vg} / \mathrm{kg}\right)$, a medium dose $\left(2 \times 10^{13} \mathrm{vg} / \mathrm{kg}\right)$ and a high dose $\left(6 \times 10^{13} \mathrm{vg} / \mathrm{kg}\right)$ [25]. The level of expressed FVIII was 12-237\%. 50 IU/dL of FVIII was maintained in six of seven patients enrolled in the highest dose group with a remarkable decrease in ABR and FVIII use. All patients in the highest dose group received corticosteroid to prevent liver toxicity. Phase 3 studies are now ongoing, and several other clinical trials have been established by different companies.

The overall results of these clinical trials of gene therapies for hemophilia A and B have been promising. Several difficulties remain to be addressed, however, including the presence of neutralizing antibodies against the AAV capsid. The overall prevalence of these antibodies is $30-50 \%$, and patients with such antibodies are ineligible for gene therapy. The vector dose for hemophilia A gene therapy is much higher than that for hemophilia B. Mild to moderate ALT elevation has been recorded in most participants, although there has been little evidence of pathological liver dysfunction. Moreover, AAV vectors are predominantly localized in the nucleus as non-replicating episomes, and therefore, transgene expression would be expected to decline over time. In this context, FVIII expression was found to be durable for 2-3 years, but the level could decline to around $5 \mathrm{IU} / \mathrm{dL}$ after eight years based on the FVIII expression model described in a Phase 2 study [26]. Gene toxicity also deserves careful consideration. Random integrations after AAV gene transduction causing insertional mutagenesis cannot be completely disregarded. The development of FVIII or FIX inhibitors also remains a clinical challenge.

The principles of future gene therapy for hemophilia may be based on overwriting the FVIII or FIX gene in hepatocytes. This genome-editing concept is believed to provide an ideal therapeutic approach for the repair of aberrant genes. This may be achieved by artificial nucleases targeting double-strand breaks (DSBs) at specific DNA sites. Several protocols have been described for this purpose, including zinc-finger nucleases (ZFNs), TAL effector nucleases (TALENs) and clustered regularly interspaced short palindromic repeats (CRISPER)/CRISPER-associated; Cas9. Recently, Ohmori et al. reported that Cas 9 can be expressed in nearly all liver parenchymal cells, and that the bleeding tendency in hemophilia B mice was improved by inserting cDNA using a knock-in technique [27]. Further studies are warranted to determine if advances with gene-editing technology of this nature has the potential for wider applicability and safer treatment eliminating the unsolved complications confronting current gene therapy using viral vectors.

\section{References}

1. Kitazawa T, Shima M. Emicizumab, a humanized bispecific antibody to coagulation factors IXa and X with a factor VIIIa-cofactor activity. Int J Hematol. 2018. https://doi.org/10.1007/s12185-0182545-9(Epub ahead of print).

2. Uchida N, Sambe T, Yoneyama K, Fukazawa N, Kawanishi T, Kobayashi S, et al. A first-in-human phase 1 study of ACE910, a novel factor VIII-mimetic bispecific antibody, in healthy subjects. Blood. 2016;127(13):1633-41.

3. Shima M, Hanabusa H, Taki M, Matsushita T, Sato T, Fukutake $\mathrm{K}$, et al. Factor VIII-mimetic function of humanized bispecific antibody in hemophilia A. N Engl J Med. 2016;374(21):2044-53.

4. Shima M, Hanabusa H, Taki M, Matsushita T, Sato T, Fukutake $\mathrm{K}$, et al. Long-term safety and efficacy of emicizumab in a phase $1 / 2$ study in patients with hemophilia A with or without inhibitors. Blood Adv. 2017;1(22):1891-9.

5. Oldenburg J, Mahlangu JN, Kim B, Schmitt C, Callaghan MU, Young G, et al. Emicizumab prophylaxis in hemophilia A with inhibitors. N Engl J Med. 2017;377(9):809-18.

6. Young G, Liesner R, Chang T, Sidonio Jr. R, Oldenburg J, Jiménez-Yuste $\mathrm{V}$ et al. A multicenter, open-label, phase 3 study of emicizumab prophylaxis in children with hemophilia A with inhibitors. Blood. 2019;2019001869. https://doi.org/10.1182/blood.

7. Mahlangu J, Oldenburg J, Paz-Priel I, Negrier C, Niggli M, Mancuso ME, et al. Emicizumab prophylaxis in patients who have hemophilia A without inhibitors. N Engl J Med. 2018;379(9):811-22.

8. Pipe S, Shima M, Lehle M, Shapiro A, Chebon S, Fukutake $\mathrm{K}$, et al. Efficacy, safety, and pharmacokinetics of emicizumab prophylaxis given every 4 weeks in people with haemophilia $\mathrm{A}$ (HAVEN 4): a multicentre, open-label, non-randomised phase 3 study. Lancet Haematol. 2019;6(6):e295-e305. https://doi. org/10.1016/S2352-3026(19)30054-7(Epub 2019 Apr 16).

9. Shima M, Nogami K, Nagami S, Yoshida S, Yoneyama K, Ishiguro A, et al. A multicentre, open-label study of emicizumab given every 2 or 4 weeks in children with severe haemophilia A without inhibitors. Haemophilia. 2019. https://doi.org/10.1111/hae.13848 (Epub ahead of print).

10. Callaghan M, Negrier C, Paz-Priel I, Chebon S, Lehle M, Mahlangu $\mathrm{K}$, et al. Emicizumab treatment is efficacious and well tolerated long term in persons with hemophilia A (PwHA) with or without FVIII inhibitors: Pooled data from four HAVEN studies. J Thromb Haemost OC 60.2. 2019.

11. Chowdary S. Anti-tissue factor pathway inhibitor (TFPI) therapy: a novel approach to the treatment of haemophilia. Int J Hematol. 2018. https://doi.org/10.1007/s12185-018-2548-6(Epub ahead of print).

12. Waters EK, Genga RM, Schwartz MC, Nelson JA, Schaub RG, Olson KA, et al. Aptamer ARC19499 mediates a procoagulant hemostatic effect by inhibiting tissue factor pathway inhibitor. Blood. 2011;117(20):5514-22.

13. Dockal M, Pachlinger R, Hartmann R, Knappe S, Sorensen B, Wong WY, et al. Biological explanation of clinically observed elevation of TFPI plasma levels after treatment with TFPI-antagonistica aptamer BAX 499. Blood. 2012;120(21):1104.

14. Chowdary P, Lethagen S, Friedrich U, et al. Safety and pharmacokinetics of anti-TFPI antibody (concizumab) in healthy 
volunteers and patients with hemophilia: a randomized first human dose trial. J Thromb Haemost. 2015;13:743-54.

15. Eichler H, Angchaisuksiri P, Kavakli K, Knoebl P, Windyga J, Jiménez-Yuste V. A randomized trial of safety, pharmacokinetics and pharmacodynamics of concizumab in people with hemophilia A. J Thromb Haemost. 2018;16(11):2184-95.

16. Eichler H, Angchaisuksiri P, Kavakli K, Knoebl P, Windyga J, Jiménez-Yuste V, Harder Delff P, Chowdary P. Concizumab restores thrombin generation potential in patients with haemophilia: Pharmacokinetic/pharmacodynamic modelling results of concizumab phase 1/1b data. Haemophilia. 2019;25(1):60-6.

17. Shapiro AD, Angchaisuksiri P, Astermark J, Benson G, Castaman G, Chowdary P, et al. Subcutaneous concizumab prophylaxis in hemophilia A and hemophilia A/B with inhibitors: phase 2 trial results. Blood. 2019. https://doi.org/10.1182/blood.2019001542 (Epub ahead of print).

18. Parng C, Singh P, Pittman DD, Wright K, Leary B, Patel-Hett $\mathrm{S}$, et al. Translational pharmacokinetic/pharmacodynamic characterization and target-mediated drug disposition modeling of an antitissue factor pathway inhibitor antibody, PF-06741086. J Pharm Sci. 2018;107(7):1995-2004.

19. Gu JM, Zhao XY, Schwarz T, Schuhmacher J, Baumann A, Ho E, et al. Mechanistic modeling of the pharmacodynamic and pharmacokinetic relationship of tissue factor pathway inhibitor neutralizing antibody (BAY 1093884) in cynomolgus monkeys. AAPS. 2017;19(4):1186-95.

20. Ohmori T. Advances in gene therapy for hemophilia: basis, current status, and future perspectives. Int J Hematol. 2018. https://doi. org/10.1007/s12185-018-2513-4(Epub ahead of print).

21. Kay MA, Manno CS, Ragni MV, Larson PJ, Couto LB, McClelland A, et al. Evidence for gene transfer and expression of factor
IX in haemophilia B patients treated with an AAV vector. Nat Genet. 2000;24:257-61.

22. Manno CS, Pierce GF, Arruda VR, Glader B, Ragni M, Rasko JJ, et al. Successful transduction of liver in hemophilia by AAV Factor IX and limitations imposed by the host immune response. Nat Med. 2006;12:342-7.

23. Nathwani AC, Reiss UM, Tuddenham EG, Rosales C, Chowdary P, McIntosh J, et al. Long-term safety and efficacy of factor IX gene therapy in hemophilia B. N Engl J Med. 2014;371:1994-2004.

24. George LA, Sullivan SK, Giermasz A, Rasko JEJ, Samelson-Jones BJ, Ducore J, et al. Hemophilia B gene therapy with a high-specific-activity factor IX variant. N Engl J Med. 2017;377:2215-27.

25. Rangarajan S, Walsh L, Lester W, Perry D, Madan B, Laffan M, et al. AAV5-factor VIII gene transfer in severe hemophilia A. N Engl J Med. 2017;377:2519-30.

26. Pasi J, Rangarajan S, Mitchell N, Lester W, Laffan M, Madan $B$ et al. First-in-human evidence of durable therapeutic efficacy and safety of AAV gene therapy over 3-years with Valoctocogene Roxaparvotec for severe haemophilia A (BMN 270-201 Study). J Thromb Haemost LB 01.2. 2019.

27. Ohmori T, Nagao Y, Mizukami H, Sakata A, Muramatsu SI, Ozawa K, et al. CRISPR/Cas9-mediated genome editing via postnatal administration of AAV vector cures haemophilia B mice. Sci Rep. 2017;7:4159.

Publisher's Note Springer Nature remains neutral with regard to jurisdictional claims in published maps and institutional affiliations. 